\title{
Beta talasemia mayor en un niño chileno
}

Los síndromes talasémicos son trastornos heredados en los cuales hay deficiencia primaria de la producción de una o más de las cadenas de globina de la hemoglobina A normal. ${ }^{1}$

La Beta talasemia es una enfermedad familiar hereditaria de carácter dominante no ligado al sexo. La producción de cadena Beta es limitada y la hemoglobina A está disminuida, existiendo una compensación parcial por las hemoglobinas que no contienen la cadena Beta; la hemoglobina A 2 y la hemoglobina $\mathbf{F}$.

La forma homocigota se manifiesta con anemia severa, retardo del crecimiento, hepatoesplenomegalia, cambios marcados en la estructura ósea como consecuencia de la proliferación intramedular del tejido eritropoyético y una dependencia de transfusiones sanguíneas. ${ }^{1}$

La Beta talasemia menor heterocigota se expresa más frecuentemente por hipocromía, microcitosis y un porcentaje elevado de hemoglobina A $2 .^{3}$ Si bien hay casos descritos con niveles relativamente elevados tanto de $\mathrm{HbF}$ como de A $2^{15}$ y aun casos con aumento de $\mathrm{HbF}$ y porcentaje normal de A $2 .^{16-17}$

En 1973 Rona y cols. publican dos casos de Sickle talasemia menor. No hemos encontrado publicaciones sobre talasemia mayor en la literatura nacional.

En este trabajo se presentan dos casos de talasemia, madre e hijo, en una familia chilena.

\section{CASO CLINICO}

P.R.R. sexo masculino, consulta por primera vez, en 1973 , a la edad de 2 años 4 meses.

Antecedentes: hijo de incesto (padre e hija). Parto normal. P.R.N., 2.800 grs. Periodo de recién nacido normal.

*Departamento de Pediatría, Hospital Clínico Regional de Concepción.

**IXepto. Hematología, Hospital Trudeau

***Depto. Hematología, Hospital Clínico, Concepción
Dres. Sergio Ceresa*, Alberto Daiber**, Exequiel González***

A los 3 meses de edad pesaba $5 \mathrm{kgr}$., desde el $5^{\circ}$ mes la madre nota palidez terrosa. Presentaba además infecciones respiratorias virales a repetición. Sin antecedentes de sangramiento. No hay antecedentes familiares de anemia hemolítica. Madre: chilena (aparentemente sana). Padre fallecido accidentalmente hace dos años (suicidio).

Es traido a nuestro hospital por palidez intensa, decaimiento y retraso psicomotor.

En el examen físico destacaban: retardo del crecimiento (talla: $74 \mathrm{cms}$, peso $10 \mathrm{kgs}$.), palidez terrosa intensa, retardo psicomotor (no se para, no camina, no habla). El aspecto de la cara demostraba prominencia frontal y de los malares, depresión del puente de la nariz, rasgos mongoloides de los ojos y prominencia del maxilar superior; cráneo natiforme, cardiomegalia con soplo sistólico mesocárdico II-IIr. El bazo estaba muy aumentado de tamaño $(6 \mathrm{~cm}$ bajo $\mathrm{el} \mathrm{re-}$ borde costal), consistencia dura; hígado, $5 \mathrm{~cm}$ (Figs. 1-2).

A los 3 dias de ingreso presentó palidez acentuada y sudoración. Los exámenes de laboratorio en esa oportunidad se expresan en la tabla 1. Dada la intensidad de la anemia se indicó una serie transfusional con lo que las condiciones del niño mejoraron notoriamente.

El estudio radiológico reveló: radiografia de cráneo: aumento del diploe crancano. Cráneo en cepillo (Fig. 3). Radiografia de huesos largos: osteoporosis. Adelgazamiento de la cortical (Fig. 4). Radiografía de tórax: gran aumento de la silueta cardíaca (Fig. 5)

En marzo de 1974 ingresó nuevamente por palidez marcada. Al examen físico destacaba gran destrucción de piezas dentarias y enorme hepatoesplenomegalia. Un hemograma demostró una hemoglobina de 3,5 grs. \% y $\mathrm{Ht} 13 \%$. La morfología de los eritrocitos no había variado con respecto a los hemogramas anteriores. Se procedió a nuevas transfusiones llevando la hemoglobina a 7 grs\%. En julio de 1974 las condiciones del niño exigieron una nueva serie transfusional.

Las características clínicas y hematológicas que presentaba el niño nos permitían plantear con fundamentos la existencia de una talasemia. Su comprobación definitiva requería, sin embargo, de estudio electroforético de hemoglobina tanto en el niño como en la madre, razón por la cual, en octubre de 1974, se mandó al paciente y a su madre al Departamento de Hematología del Hospital Trudeau.

Los estudios realizados en dicho hospital demostraron en el niño una $\mathrm{HbF}$ de $51 \%$ (no había recibido transfusiones en los últimos 3 meses), $\mathrm{Hb}$ A2 casi ausente. El hemograma demostró anemia intensa con rasgos talasémicos típicos. Ferremia $276 \mu \mathrm{g} \%$ (normal hasta 150). Resistencia globular osmótica aumentada. Durante su hospitalización se 
Tabla 1

FXA MENES PRR DE LABORATORIO AL INGRESO

Recuento herici :ligrco

Eritrocitos

Hemoglobina

Hematocrito

Eritroblastos

Anisocitosis ++++

Poiquilocitosis $t++$

$$
\begin{gathered}
2.500 .000 \times \mathrm{mm}^{3} \\
4,8 \mathrm{grs} \% \\
17 \% \\
15 \%
\end{gathered}
$$

Microcitosis +++

Dianocitos ++

Negativo

Aumentada

Basal: $200 \mu \mathrm{g} \%$

Capacidad total de combinación: $300 \mu \mathrm{g} \%$

Saturación de la transferrina: $66,8 \%$

$62 \%$

Total: 2,08 mgrs $\%$

No conjugada: 1,22 mgrs $\%$

Eritroblastos con gránulos de glicógeno administraron transfusiones, observándose una franca mejoria aunque permaneciendo su alteración psicomotora. La esplenomegalia en el momento de su alta era de 8 crns

Con oportunidad de este viaje a Santiago, se aprovechó de estudiar a la madre, obteniéndose los siguientes resultados: M.R.G., 20 años, sin antecedentes mórbidos. Examen físico negativo. Hemograma normal. Ferremia $135 \mu \mathrm{g} \%$. Resistencia globular osmótica aumentada. El estudio electroforético de $\mathrm{Hb}$ demostró una $\mathrm{HbF}$ normal y $\mathrm{Hb}$ A 2 elevada (tabla 2)

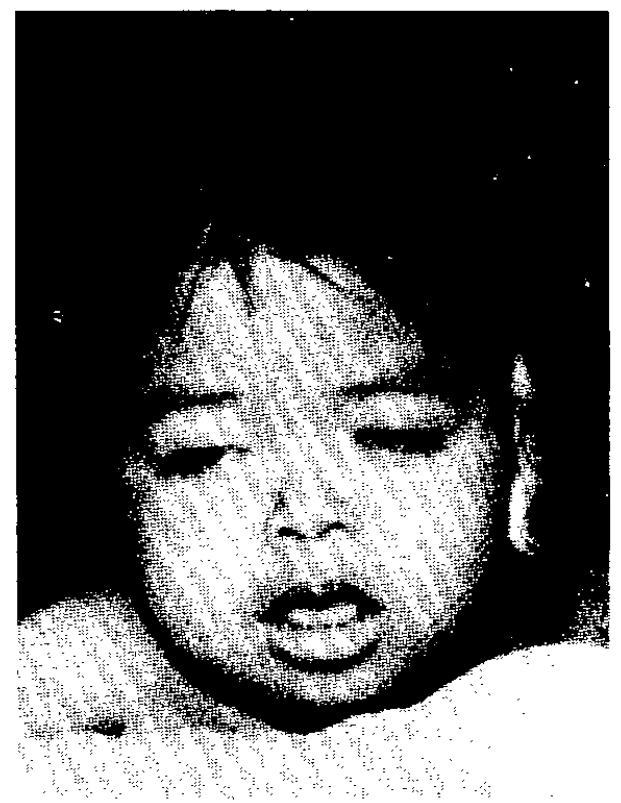

Fig. 1

Rasgos talasémicos típicos en P.R.R.
Tabla 2

ESTUDIO HEMATOLOGICO EN MRG (madre) y PRR (hijo)

\begin{tabular}{lcc}
\hline Hematics (millones) & $\mathbf{4 , 5 1}$ & 2,01 \\
Hbgr\% & 12,6 & 4,3 \\
Hto & 40,0 & 14,5 \\
CMHC & 31 & 29 \\
Hb F\% & 1,2 & 51,0 \\
HbA 2 & 1,5 & 8,0 \\
Reticulocitos & 1,5 & $\mathbf{8 , 0}$ \\
Sickling & $(-)$ & $(-)$ \\
\hline
\end{tabular}

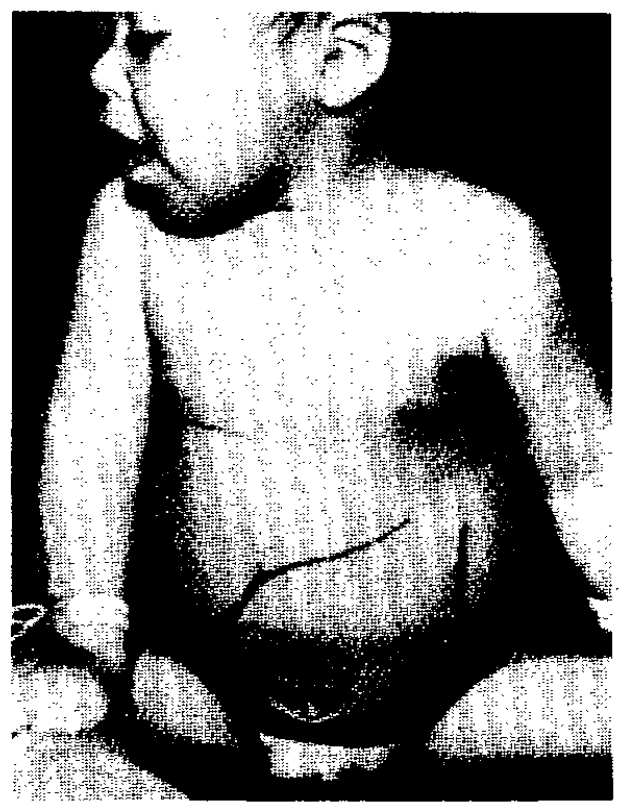

Fig. 2

Gran hépato y esplenomegalia en P.R.R. 


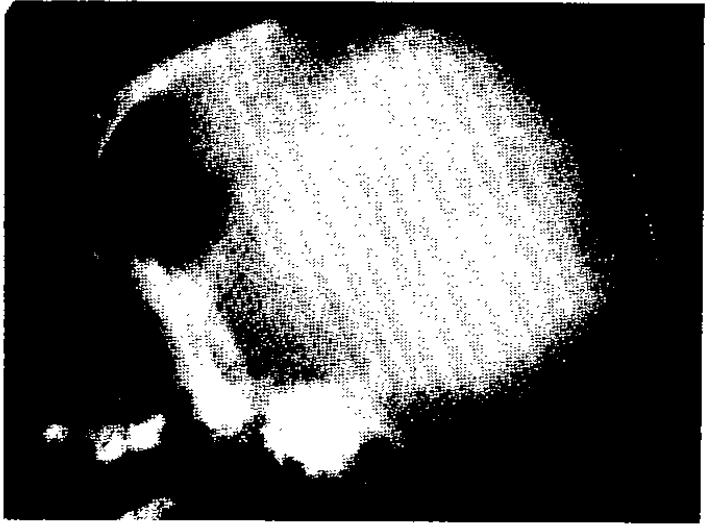

Fig. 3

Radiografia de cráneo: aumento del diploe craneano. Cránen en cepillo.

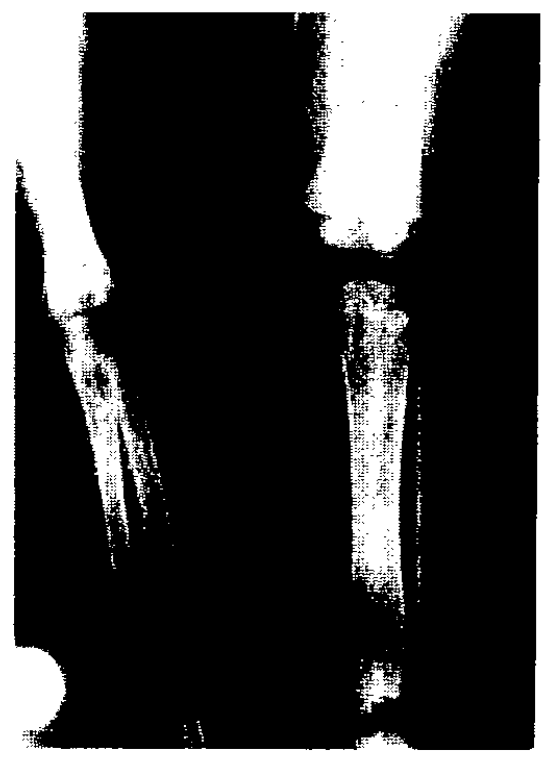

Fig. 4

Radiografia de huesos largos: usteoporoszs. Adtrgazamiento de la cortical
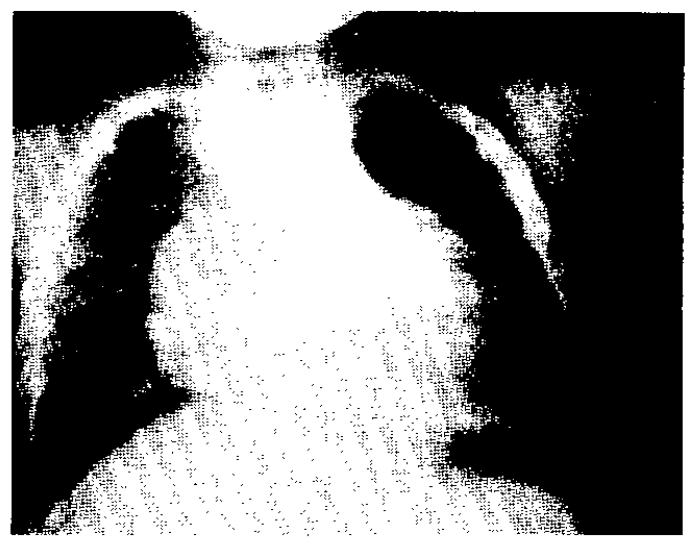

Fig. 5

Radiografia de tórax: gran anmento de la silueta cardiaca.
El estudio de este niño y de su madre, ha demostrado que se trata de talasemias de tipo Beta. Weatheral y otros autores han hecho notar que uno de los hallazgos más característicos, en los portadores heterocigotos de la Beta talasemia, es el aumento de la $\mathrm{Hb} \mathrm{A2}$, con o sin aumento de la HbF. En la madre se encontró aumento claro de A2 y resistencia globular osmótica aumentada, lo que confirma que ella es portadora de una Beta talasemia (heterocigota inaparente) a pesar de no tener el rasgo bien desarrollado en sus hematíes y de no presentar anemia. En el hijo, en cambio, se encontró una anemia intensísima, hemolítica y con todas las características del rasgo telasémico: anisocitosis hipocroma con dianocitos, poiquilocitocitos y punteado basófilo; además, gran aumento de HbF (51\%) (los niños a los 3 meses de edad tienen un máximo de $2 \%$ ) y casi ausencia de Hb A2. Todo esto certifica la naturaleza homocigota de su talasemia. El antecedente de haber sido concebido por incesto (padre e hija) nos permite asegurar que su padre (y abuelo a la vez) debió haber sido también un portador heterocigoto.

El tratamiento de la anemia de Cooley aún consiste en transfusiones repetidas, tratando de conservar la hemoglobina sobre 8 a 9 $\operatorname{grs} \% .{ }^{13}$ La transfusión a estas cifras altas exige, desde luego, un requerimiento transfusional que inevitablemente, contribuye en forma importante a producir una sobrecarga de fierro en el organismo. El uso de agentes de quelación, entre ellos la desferroxiamina, deben ser empleados para eliminar el exceso de fierro parenteral. ${ }^{\ominus}$ La esplenectomía debe ser planteada cuando aumenta la necesidad de transfusiones y cuando hay aumento masivo del volumen del bazo. Su indicación debe ser cuidadosa por la alta frecuencia de infección después de ella y la mayor susceptibilidad a enfermedades cardíacas y renales que dependen de infecciones estreptocócicas en los talasémicos. ${ }^{14}$ Actualmente se sabe, por los trabajos de Nathan y Fessas, que en la Beta talasemia las cadenas Alfa libres se acumulan en los glóbulos rojos y eritroblastos formando cuerpos de inclusión, lo que origina aumento de la rigidez del eritroblasto, daño de la membrana y lisis ulterior. Un tratamiento más racional debería llevarnos a la supresión de cadenas Alfa en 
eritroblastos humanos o a tratar de aumentar la producción de cadenas Gamma, ya que con ello se favorecería la producción de $\mathrm{HbF}$ cuya concentración, aun en tasas elevadas, no es deletérea para el glóbulo rojo. Estos alcances están aún en etapas de investigación.

El pronośtico en este niño es muy malo. El daño provocado por la anemia intensa a que ha estado sometido parece irreparable; a ello se suma el efecto deletéreo del exceso de fierro en su organismo y que to llevará a la hemocromatosis clásica. « pesar de esto último, el niño debe ser sometido a transfusiones regulares, para mantener la hemoglobina a un nivel adecuado. Como se dijo anteriormente, el empleo continuado de quelantes del tipo de la desferroxiamina estaría indicado para disminuir la sobrecarga de fierro secundaria al proceso hemolítico y exagerada con las transfusiones. Si su administración continuada durante años no da resultados adecuados, ello puede ser atribuido, en parte, al hecho que la instalación de la terapia quelante se ha iniciado tardíamente.

Queremos destacar, finalmente, que la talasemia en Chile no es una afección rara como se ha descrito en otros trabajos de la literatura nacional. ${ }^{6}$ Estudios recientes realizados en el Departamento de Hematología del Hospital Trudeau están demostrando que, muy por el contrario, el gen talasémico en su forma heterocigota es muy frecuente, predominando, por cierto, los portadores inaparentes. La confirmación de cada caso requiere de técnicas delicadas que sólo algunos centros pueden realizar; ello explicaría la falsa imagen que se ha creado en torno a talasemia en el genoma del chileno.

\section{RESUMEN}

Se comunican dos casos de talasemia en una familia chilena. Los hallazgos clínicos y de laboratorio son similares a los que se describen en la literatura. Se analiza el tratamiento actual de la talasemia mayor y sus perspectivas a futuro. Se destaca que la baja incidencia de esta enfermedad en nuestro medio se debe a que no se cuentan con los medios adecuados para su diagnóstico en nuestros centros hospitalarios.

\section{REFERENCIAS}

1 Weatherall, D.J. The Thalassemia Syndromes. Philadelphia, Fa Davis, 1965.

2 Schuartz Elıas, M.D New Engl. J. Med., 281:13271332, 1969.

3 Vathan David, M.D. New Engl. J. Med., 281:1363$1364,1969$.

4 Schuartz Eltas. M.D. Clínicas Pediátricas de N.A., Nov. 1972.

5 Daber, $A$ y $H$. de la Ciuadra. Rev. Méd. Chile, 96: 486, 1968.

6 Rona, $R$ y cols. Rev. Méd. Chile, 96:237, 1973.

7 Guzmán, ( $\therefore$ y cols. IX Congreso de Hematología. (Relato)

8 Nathan, D.G. y R.B. Clenn. Amer. J. Med., 41:815, 1966.

9 Diwany, M. y cols. Arch. Dis. Child., 43:340, 1968.

10 Fessas, Ph. Blood, 27:21, 1963.

11 Smith, C.H Hematología Pediátrica, Salvat, Barcelona, 1969, 314-387.

12 Daiber, A. y cols. Hemoglobinopatías en Chile. (Por publicarse.)

13 Wolf, J.A. and K.H. Luce. Ann. N.Y. Acad. Sci, $5: 423,1969$.

14 Wasi, P. Lancet, 1:949, 1971.

15 Schokker, R.C. and L.N. Went. Nature (London), 239:44-46, 1966.

16 Malamos, B. and P. Fessas. Brit. J. Haemat, 8:514, 1962.

17 Gabizita, T.G. and D.G. Nathan New Engl. J. Med., 27\%:1212-1217, 1964. 